\title{
Verwundbare Banken
}

\section{Michael Kogler}

\section{Relevanz}

Die Verflochtenheit der Banken über gegenseitige Kreditbeziehungen und über den Wertpapierhandel begünstigt das Entstehen systemischer Risiken. Werden Interbankenkredite notleidend, geraten andere Banken in Schwierigkeiten. Tätigen Banken umfangreiche Notverkäufe, kann dies einen Preisverfall auslösen und auch die Vermögenswerte anderer Institute reduzieren. Der Beitrag einzelner Banken zum systemischen Risiko kann sehr unterschiedlich sein und hängt bei weitem nicht nur von ihrer Größe ab. Um Krisenrobustheit und Wachstum gleichermaßen zu stärken, sollte nicht die Verschuldung der Banken und damit die Refinanzierung ihrer Kreditvergabe eingeschränkt, sondern mehr Eigenkapital gebildet werden. Dabei wäre es wichtig, gezielt Banken, die stark zum systemischen Risiko beitragen, mit mehr Eigenkapital auszustatten und generell die Bedingungen für die Eigenkapitalbildung zu verbessern.

Christian Keuschnigg

\section{Quelle}

Der nachfolgende Text ist eine Zusammenfassung von: Greenwood, Robin, Augustin Landier und David Thesmar (2015), Vulnerable Banks, Journal of Financial Economics 115, 471-485.

M. Kogler $(\bowtie)$

Universität St. Gallen, St. Gallen, Schweiz

E-Mail: Michael.Kogler@unisg.ch 
Die Finanzkrise zeigt, dass man Bankrisiken nicht isoliert betrachten kann, da sich die Krise einer einzelnen Bank mitunter rasch auf andere ausbreitet. Diese Ansteckungsgefahren motivierten massive Interventionen von Politik und Zentralbanken während der Krise (z. B. Liquiditätshilfen, Rekapitalisierungen oder Verstaatlichungen). Das Phänomen der Ansteckung, oft auch als systemisches Risiko bezeichnet, macht Banken besonders verwundbar und unterscheidet sie grundsätzlich von anderen Unternehmen. Dies begründet weitergehende Regulierung und Interventionen in diesem Sektor. Man unterscheidet zwischen zwei verschiedenen Ansteckungsmechanismen: Erstens sind Banken über gegenseitige Interbankkredite, welche in erster Linie der kurzfristigen Refinanzierung dienen, miteinander verbunden. Die Krise einer Bank führt zum Kreditausfall und zu Verlusten bei anderen Banken, wodurch diese ebenfalls in Schwierigkeiten geraten können. Zweitens sehen sich Banken mit Liquiditätsproblemen oder Verlusten infolge von Kreditausfällen oder Schwankungen von Wertpapierpreisen oft gezwungen, rasch Vermögenswerte zu veräußern. Mit den Verkaufserlösen können sie ihre Verbindlichkeiten reduzieren, bis ihr Eigenkapital ausreicht, die Kapitalvorschriften zu erfüllen (sog. Deleveraging). Die Alternative, zusätzliches Eigenkapital aufzunehmen, ist in einer Krisensituation oft schwierig und teuer. Für viele Vermögenswerte von Banken (z. B. Kredite, Sicherheiten) gibt es nur wenige Käufer, sodass kein liquider Markt existiert. Deshalb kann der Verkauf zu einem raschen Preisverfall führen. Davon sind auch andere Banken, welche dieselben Vermögenswerte in ihrer Bilanz halten, betroffen; sie müssen ihre Positionen wertberichtigen oder realisieren Verluste. Es kommt zur Ansteckung, da krisenbedingte Verkäufe einer Bank Verluste bei anderen Banken verursachen, sodass diese möglicherweise auch Notverkäufe durchführen müssen. Diese Abwärtsspirale - sogenannte Fire Sales - beschreibt die rasche, oft unkontrollierbare Ausbreitung eines Schocks über Assetpreise.

\section{Ansteckungsgefahren zwischen Banken bestehen durch Interbankkre- dite und eine Abwärtsspirale von Preisen illiquider Vermögenswerte (Fire Sales).}

Greenwood, Landier und Thesmar (2015) analysieren dieses Phänomen und entwickeln ein strukturelles Modell, welches die zentralen Mechanismen illustriert: Aufgrund eines Preiseinbruchs bei bestimmten Wertpapierkategorien erleiden Banken Verluste, die ihr Eigenkapital schmälern. Sie liquidieren Teile ihrer Aktiva, um mit den Erlösen Verbindlichkeiten zu verringern und die Eigenkapitalquote wiederherzustellen. Solange die Vermögenswerte illiquid sind, führen Verkäufe zu einem Preisverfall und zu Verlusten bei anderen Banken. Solche Verkäufe können alle Vermögenswerte umfassen, nicht nur jene, welche den 
ursprünglichen Preisverfall erlitten. Der Einbruch von Staatsanleihenpreise kann beispielsweise auch zu einem Rückgang der Aktienpreise führen, sobald Banken darauf mit dem Verkauf von Aktien reagieren. Dieser Ansatz ermöglicht es, sowohl die Verwundbarkeit einzelner Banken gegenüber systemweitem Deleveraging als auch ihre Systemrelevanz (d. h. ihren Beitrag zu den Gesamtverlusten) zu quantifizieren. Damit werden die Auswirkungen eines Schuldenschnitts auf Staatsanleihen von GIIPS-Staaten und verschiedene Politikoptionen simuliert. Der zentrale Beitrag dieses Papiers liegt in der Entwicklung eines Ansatzes, welcher die wichtigsten Ansteckungsmechanismen illustriert und zur Simulation verschiedener Szenarien dient, und in der Quantifizierung von Systemrelevanz und Verwundbarkeit einzelner Banken.

Die individuelle Verwundbarkeit quantifiziert das Exposure einer Bank gegenüber einem plötzlichen Verfall von Assetpreisen gemessen an ihrem Eigenkapital. Die direkte Verwundbarkeit umfasst die Verluste, die unmittelbar aufgrund dieses Schocks auftreten (z. B. Wertberichtigungen der betroffenen Bilanzpositionen), die indirekte Verwundbarkeit jene weiteren Verluste, die aufgrund von Fire Sales entstehen und prinzipiell alle Vermögenswerte betreffen können. Die indirekte Verwundbarkeit hängt vom Verschuldungsgrad sowie vom Umfang jener Vermögenswerte, die stark von Fire Sales betroffen sind, in der Bankbilanz ab: Eine schwach kapitalisierte Bank mit vielen Assets, die illiquide sind und nach dem Preiseinbruch von zahlreichen Banken verkauft werden, gilt als besonders verwundbar.

Die Systemrelevanz hingegen beschreibt den Effekt des Deleveragings und der Notverkäufe einer bestimmten Bank auf das Eigenkapital des gesamten Bankensektors: Welcher Anteil des Eigenkapitals der Banken wird durch die Notverkäufe einer bestimmten Bank aufgezehrt? Eine Bank gilt dann als systemrelevant, wenn ihr Deleveraging einen starken Rückgang der Assetpreise mit hohen Verlusten für andere Banken auslöst. Die Systemrelevanz hängt von mehreren Faktoren ab: Größe und Verschuldungsgrad der Bank sowie die Höhe des Preiseinbruchs bestimmen den Umfang ihres Deleveragings. Die Verknüpfungen mit anderen Banken sind zentral: Eine Bank ist stark mit anderen verknüpft, wenn sie über viele illiquide Assets verfügt, die in großem Umfang von anderen Banken gehalten werden. Dann hat ihr Deleveraging einen besonders starken Effekt. Die Summe der individuellen Beiträge der Banken zum aggregierten Eigenkapitalverlust entspricht schließlich den Verlusten aller Banken durch Fire Sales. Diese Größe quantifiziert das systemische Risiko des Bankensektors.

Grösse, Verschuldungsgrad, Verknüpfung mit anderen Banken und die Verwundbarkeit gegenüber dem Preisverfall bestimmen die Systemrelevanz einer Bank. 
Greenwood u. a. (2015) simulieren die Auswirkungen eines Schuldenschnitts auf Staatsanleihen der GIIPS-Staaten - Griechenland, Italien, Irland, Portugal und Spanien - in Höhe von 50 \%. Dazu verwenden sie Bilanzdaten der 90 größten europäischen Banken aus dem EBA Stresstest 2011, als die Banken stärker als heute in den betroffenen Staaten investiert waren. Eine typische Bankbilanz besteht aus bis zu 42 Assetkategorien, die alle von Fire Sales betroffen sein können. Die wichtigsten Kategorien sind Unternehmenskredite (29\% der gesamten Bankaktiva), Hypotheken (20\%) und Staatsanleihen (13\%). Basierend auf diesen Daten wird die Verwundbarkeit jeder einzelnen Bank berechnet: Im Durchschnitt verursacht der Schuldenschnitt unmittelbare Wertberichtigungen und Verluste von $111 \%$ des Eigenkapitals. Diese lösen Notverkäufe und einen Preisverfall mit zusätzlichen Verlusten im Umfang von $302 \%$ aus. Der indirekte Effekt der Fire Sales ist also substanziell höher als der direkte Effekt des Schuldenschnitts. Zudem sind jene Banken, die am stärksten vom Schuldenschnitt betroffen sind, nicht identisch mit jenen, die am stärksten unter den Notverkäufen leiden: Bei den Österreichischen Volksbanken, zum Beispiel, führen diese zu Verlusten von $483 \%$ ihres Eigenkapitals wogegen der direkte Verlust des Schuldenschnitts nur $20 \%$ des Eigenkapitals beträgt. Diese Diskrepanz lässt sich dadurch erklären, dass die Volksbank vergleichsweise wenige betroffene Staatsanleihen, jedoch viele illiquide Vermögenswerte, die in der Folge stark unter Fire Sales leiden, in ihrer Bilanz hält.

Ebenso kann man die Systemrelevanz einer Bank, der Beitrag ihres Deleveragings zu den aggregierten Verlusten, berechnen: Der Schuldenschnitt führt zu direkten Verlusten im Umfang von 381 Mrd. € bzw. rund $40 \%$ des gesamten Eigenkapitals des Bankensektors und löst Notverkäufe mit Verlusten im Umfang von $245 \%$ des gesamten Eigenkapitals aus. Die aggregierten Verluste durch Fire Sales sind mehr als sechsmal höher als die unmittelbaren Verluste infolge des Schuldenschnitts. Erstere können in die Beiträge durch das Deleveraging jeder einzelnen Bank zerlegt werden: Die höchsten Verluste entstehen aufgrund der Notverkäufe der Banco Santander (21\% des aggregierten Eigenkapitals), der Unicredit (19\%) und der Intensa SanPaolo (19\%), welche daher als die drei systemrelevantesten Banken gelten. Die Systemrelevanz besteht aus drei Komponenten: Größe, Umfang der Fire Sales und Verknüpfungen mit anderen Banken. Eine große Bank ist auch systemrelevant, wenn sie aufgrund des Schuldenschnitts ein starkes Deleveraging durchführt (z. B. weil sie viele betroffene Staatsanleihen hält und wenig Eigenkapital hat) und dieses vorwiegend den Verkauf illiquider Vermögenswerte, die von vielen anderen Banken gehalten werden, umfasst. Diese Verknüpfungseffekte erklären, weshalb die größte Bank der EU, die $H S B C$, nicht 
zu den zehn systemrelevantesten Banken gehört, Banken mit bestenfalls durchschnittlicher Größe wie die Monte Dei Paschi di Siena oder die Caja de Ahorros $Y$ Pensiones de Barcelona hingegen schon.

Die Eigenkapitalverluste des Bankensektors durch Fire Sales sind mehr als sechsmal so hoch wie die direkten Verluste durch einen Schuldenschnitt.

Basierend auf diesem Szenario simulieren Greenwood u. a. (2015) verschiedene wirtschaftspolitische Maßnahmen mit dem Ziel, das systemische Risiko (d. h., die aggregierten Verluste durch Fire Sales) zu verringern. Eine teilweise Renationalisierung der GIIPS-Staatsschulden kann das systemische Risiko um acht Prozent verringern. Dieses Ergebnis hängt allerdings stark von der Tatsache ab, dass Banken in den GIIPS-Staaten besser kapitalisiert sind, sodass der Schuldenschnitt bei ihnen ein geringeres Deleveraging auslöst als bei den schwächer kapitalisierten Banken in nicht GIIPS-Staaten. Die Renationalisierung verlagert das Exposure zu den besser kapitalisierten Banken, was den Umfang der Notverkäufe und das systemische Risiko reduziert. Die oft diskutierte Idee von Eurobonds wird so abgebildet, dass jede Bank dasselbe, diversifizierte Portfolio aller europäischen Staatsanleihen hält. Dies erhöht allerdings die Verwundbarkeit schwächer kapitalisierter nicht GIIPS Banken, welche dadurch mehr GIIPS- und weniger deutsche Staatsanleihen halten, und erhöht das systemische Risiko um acht Prozent.

Eine spürbare Reduktion des systemischen Risikos kann durch eine Begrenzung der Verschuldung erreicht werden: Zum Beispiel verringert ein maximaler Verschuldungsgrad von 15 - dies entspricht einer Eigenkapitalquote von knapp sieben Prozent - das systemische Risiko um $28 \%$. Allerdings erfordert dies eine drastische Erhöhung des aggregierten Eigenkapitals um 480 Mrd. €. Einen ähnlichen Effekt kann man wesentlich effizienter erreichen, indem gezielt systemrelevante Banken rekapitalisiert werden: Eine gezielte Rekapitalisierung im Umfang von 200 Mrd. € führt zu einer vergleichbaren Reduktion des systemischen Risikos $(26 \%)$ wie die erwähnte Begrenzung des Verschuldungsgrades, welche allerdings den Einsatz von mehr als doppelt so viel Eigenkapital erfordert.

Die Begrenzung des Verschuldungsgrades und die gezielte Rekapitalisierung systemrelevanter Banken verringern das systemische Risiko am stärksten. 
Zentrales Ergebnis der Analyse von Greenwood u. a. (2015) ist die quantitative Relevanz von Fire Sales: Die aggregierten Eigenkapitalverluste durch Fire Sales infolge eines Schuldenschnitts auf GIIPS-Anleihen sind mehr als sechsmal so hoch wie die unmittelbaren Verluste durch den Schuldenschnitt selbst. Dies unterstreicht die Wichtigkeit von Ansteckungseffekten, welche Banken besonders verwundbar machen. Ihre Analyse zeigt, dass der Beitrag einer Bank zu den aggregierten Verlusten, ihre Systemrelevanz, nicht nur von Größe, Verschuldungsgrad und Umfang des Preisverfalls sondern ganz besonders von Verknüpfungen mit anderen Banken, die durch das Halten derselben, illiquiden Vermögenswerte entstehen, abhängt. Simulationen zeigen, dass eine gezielte Rekapitalisierung systemrelevanter Banken das geeignetste Instrument darstellt, das systemische Risiko zu reduzieren.

Open Access Dieses Kapitel wird unter der Creative Commons Namensnennung 4.0 International Lizenz (http://creativecommons.org/licenses/by/4.0/deed.de) veröffentlicht, welche die Nutzung, Vervielfältigung, Bearbeitung, Verbreitung und Wiedergabe in jeglichem Medium und Format erlaubt, sofern Sie den/die ursprünglichen Autor(en) und die Quelle ordnungsgemäß nennen, einen Link zur Creative Commons Lizenz beifügen und angeben, ob Änderungen vorgenommen wurden.

Die in diesem Kapitel enthaltenen Bilder und sonstiges Drittmaterial unterliegen ebenfalls der genannten Creative Commons Lizenz, sofern sich aus der Abbildungslegende nichts anderes ergibt. Sofern das betreffende Material nicht unter der genannten Creative Commons Lizenz steht und die betreffende Handlung nicht nach gesetzlichen Vorschriften erlaubt ist, ist für die oben aufgeführten Weiterverwendungen des Materials die Einwilligung des jeweiligen Rechteinhabers einzuholen.

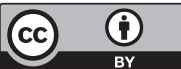

